Roberto Moreno-Díaz Bruno Buchberger José-Luis Freire (Eds.)

\title{
Computer Aided Systems Theory - EUROCAST 2001
}

A Selection of Papers from the 8th International Workshop on Computer Aided Systems Theory

Las Palmas de Gran Canaria, Spain, February 19-23, 2001 Revised Papers 


\title{
Optimizing Organizational Structure: A Hausdorff Benchmark for Complex Social Systems
}

\author{
Markus Schwaninger \\ Institute of Management, University of St. Gallen, \\ Dufourstrasse 48, CH-9000 St. Gallen, Switzerland \\ Markus. Schwaningereunisg.ch
}

\begin{abstract}
The search of optimal structures for social organizations has been an ongoing concern of management science, but reliable answers have not been given. Optimization theory has provided solutions, but these have always addressed very specific organizational problems, not the issue of overall optimality in terms of the fitness of the organization as a whole. In this paper, a new approach, grounded in system theory is suggested for assessing and ultimately also for pursuing such a general optimality of organizational designs. It is proposed that the optimal fractal dimensionality of organizations is equivalent to that of living organisms. This hypothesis is submitted to and corroborated by a first test. Herewith, a new path to a theory-based design of optimal organizations has been opened up, the implications of which for the methodology of organization design may be substantial.
\end{abstract}

\section{The Question and Traditional Ways of Dealing with It}

The question "Are there optimal structures for organizations?" has recurrently beer posed in this general form. The answers have varied, but to date the tenor has been "No, there is no generally optimal structure. Probably there is an optimal structure fo: each organization. But we are still looking for a solid theory to ascertain that."

In the past, the question of organizational optimality has been considered discussi ble only in very specific and therefore limited contexts. The more famous example: are linked with the optimization of organizational processes, e.g. routing problems resource allocation problems, and control problems. Applications of this type havi resulted in substantial improvements as far as the economic use of scarce resources i concerned. The types of problems of enduring structures, which have been studied it terms of optimization, are different:

- Single-objective optimization: Under this title the classical types of optimizatios can be subsumed. A typical case is the question of the optimal span of control it a hierarchy of agents with largely uniform tasks (e.g., the optimal number 0 salespersons in service centers for a market).

- Multi-objective, multi-parameter and multi-level optimization: Large and com plex logistic systems may call for "multiple-issue" solutions which allow for a overall optimum, taking into account different objectives at the same time (e.g., 
postal system where cost, time and ecological performance may be the preeminent criteria, and where a structure of distributive centers with several levels may be considered). Solutions for this type of problem may involve multiobjective, multi-parameter and multi-level optimization in a combined mode.

It must be noted that only a subset of the optimization techniques can provide "optimal solutions" in the strict sense of the word. Given the "curse of dimensionality" most of the more complex solutions mentioned are numerical and only approximate optimality. In other words, it is known that these solutions are close, often very close to the theoretical optimum, although that theoretical optimum remains unknown. The exact boundary between the cases with "optimum" solutions and those with "close to optimum" solutions is given by the theoretical bound of feasible computation, which establishes the physical limits of analysis. Essentially, the use of heuristic methods is motivated by the question as to whether the decision problems which are part of these optimization problems are "NP-complete". This means, to put it in a nutshell, that an exact solution of a given optimization problem is only possible if one can construct a polynomial algorithm which can solve all the decision problems of the complexity class in focus [cf. 10,28].

Another approach to the search for optimality has been multidimensional structuring. In principle, optimization can be applied to multi-dimensional problems, as has been said: Multi-objective optimization is a case in point. When we talk about "multidimensional organization" we mean something different: We indicate the simultaneous structuring of an organization according to different structuring criteria. Even though multidimensionality has contributed substantially to absorbing complexity, the question of an optimal degree of dimensionality remains open. In fact, it has barely been addressed.

To deal with this very issue, I have taken an approach, which is very much in the tradition of system theory and cybernetics: The search for an isomorphism, in other words, an invariant structural feature, which is relevant in different contexts. Such isomorphisms allow mapping systems from different domains of reality onto one and the same model. For this purpose I have reverted to the natural sciences.

Systems theorists and cybemeticians have traditionally leveraged knowledge originating from biology for the domain of social systems (e.g., Miller, Pask and Beer). Also, several of the founding fathers of Systems Theory were trained biologists: The Society for General Systems Research was founded in 1954 by Ludwig von Bertalanffy, Ralph Gerard (both biologists), Anatol Rapoport (a mathematician, who had applied mathematics to biology) and Kenneth Boulding (an economist). Rashevsky and Rapoport spearheaded the application of mathematical modeling to both biological, and social relationships. Finally, General Systems Theory has also identified structural similarities which extend beyond mere comparisons of the social and biological domains. These also include analogous situations, e.g. in chemistry and physics, where such analogies have also been formalized mathematically, in the sense that practically the same mathematical model may be employed to express theories widely disparate in content [cf. 18, 19].

Many of the loans from biology taken by social scientists have been at the level of analogy, - stimulating thought, but not leading all the way to rigorously formulated theory. There have at least been two notorious exceptions: 
1.) James Grier Miller's Living Systems Theory (LST): Miller identified 19 subsystems which make up a living system, and which are invariant across a wide spectrum of organized wholes, from the living cell to a society [17]. LST originated in the biological studies of Miller, who by training is a medical doctor.

2.) Stafford Beer's Viable Systems Model (VSM): Beer discerned a set of control functions, and their interrelationships, which are the necessary and sufficient preconditions for the viability of any human or social system. This model is based on an isomorphic mapping originating from studies of the structure of the human central nervous system. A crucial feature of the VSM is that it introduces the notion of recursiveness, in that the viability of social systems is grounded in the recursive existence and functioning of the system of (self) control it specifies $[4,5,6]$.

Both of these models are outstanding in their originality They have triggered numerous studies and applications in the realms of organization, society, and engineering. They both address the preconditions for viability, but not the issue of the optimality of structures.'

\section{Idea for an Innovative Approach and Hypothesis}

If we ask "What dimensionality is optimal?", current organization theory itself cannot provide us with satisfactory answers. Again, we have to take recourse to the natural sciences, and once more biology appears to be a reliable source of knowledge.

From biology we know that living organisms (plants, animals, humans) are structured in a fractal mode: Their metabolism, breathing, blood circulation and other vital functions are optimized by these fractal structures. Several research teams, in Germany, one of them at the University of Giessen, headed by Manfred Sernetz, have studied these structures. They have shown why and how this "strategy of fractal organization" entails the living organisms' functional superiority as compared with manmade bio-reactors.

These bio-reactors require continuous stirring to bring about the turbulence of the liquid necessary for higher efficiency of the chemical reaction, which can only be induced by high-energy input. The organism of an animal or human produces catalytic processes similar to those of a bio-reactor. However, there is no need for stirring; what the pump-function of the heart induces is just a laminar blood flow, enabled by a much lower energy level. Even so, the mixture of the liquid phase (blood) and the stationary phase (tissue) are achieved in an optimal way, with a result that a continuously stirred tank reactor (CSTR) could only mimic at enormously high cost. This stupendous superiority of the natural "reactor" is largely due to the fractal structure of the living organism.

A fractal structure is one where the principle of organization is applied in an iterative mode. Consequently the parts show the same structure as the whole; the organization is self-similar.

Fractal structure is a special case of recursive structure. However, the term "fractal" adverts to a specific notion: It derives from the Latin participle "fractus" - broken,

${ }^{1}$ A comparative evaluation of these two models is yet to be accomplished. 
which in this case denotes that the dimensionality of the object under study is usually to be expressed by a broken number, not by 1,2 or 3. A dimensionality of one would be applicable to a line; two corresponds to an area and three to a sphere. Certain aspects of self-similarity cannot be described adequately by means of the classical measures, length, surface and volume.

For example, the curve of Koch's snow flake, a classical fractal, has a finite surface but an infinite length. Also, the mathematical idealization of a tree of blood vessels with infinitely thin branches has a volume of zero although it reaches every single point inside the organ it feeds [25].

My idea was the following: If the fractal dimensionality of living organisms is optimal (as proposed by biology; [cf. 25]), and if optimal structures are invariant across different domains of reality (as claimed by systems theory; [cf. 17, 4, 5, 6]), then the benchmark for optimal structures should be the size of that dimensionality.

Friedrich Hausdorff, in his landmark article on dimension and external measure [11] defined a measure for the size of objects independent of the resolution of the measurement, ' $e$ '. The Hausdorff dimension is a very general measure for the dimensionality of objects, applicable to any shape ${ }^{2}$.

The dimension of a fractal can be ascertained by determining its conventional measures with the help of increasingly fine yardsticks, and by ascertaining how much the measures grow as a function of this refinement. Real biological objects can be measured in this way, whereby their fractal dimension can be determined, with the help of different methods (e.g. Mass-radius-analysis). Biometric studies have ascertained that the metabolic activity of living organisms follows a law of power, expressed by the following formula, which has been derived from empirical evidence [after 24, 26, 14]:

$$
M=L^{D}, \text { where } 2,2>\mathrm{D}>2,3 .
$$

$\mathrm{M}$ is the metabolic activity, measured as the organism's throughput in Joule per second, and $\mathrm{L}$ is the length of the organism. $\mathrm{D}$ is the fractal dimensionality of the organism.

On the basis of measures of multiple species, Sernetz and his team ascertained an allometric exponent of $b=0,74$, measuring the progression of throughput in Joule per second, as a function of body volume in litres. Expressed by the length instead of the volume, the applicable exponent is $D=3 b=2,22$, which denotes the growth of the metabolic rate as a function of the length of an organism. On the assumption that the extant living organisms are optimally structured, Sernetz concludes that an optimally built organism must be 2,22-dimensional [25]. In other words, according to this theory, for optimally built organisms the law of power must be:

$$
M=L^{2,22} \text {. }
$$

If we continue taking the functioning of living organisms as a role-model for social organizations, we may be on the threshold to a further development of organizational theory.

We know that social organizations, in terms of communication and information, show properties, which are in a certain sense identical to those of living organisms.

\footnotetext{
${ }^{2}$ The dimensionality is usually, albeit not necessarily fractal.
} 
Even though there are fundamental differences - social organizations are constituted by autonomous agents with their own goals, preferences and values [1] - they resemble living organisms to a high degree. If we take the internal transfer of knowledge as a case in point, we can suppose that this is, in principle, the most important mechanism of adaptation. This can at least be derived from studies made during the last decade [cf. 12 and literature quoted therein].

If the structural laws governing the behavior of social organizations and living organisms are the same, and there is a large body of evidence indicating that they are, then we can make use of a powerful isomorphy (i.e., structural invariance):

Similarly to an optimally built organism, an optimally conceived organization should exhibit a dimensionality of about 2,2 to 2,3 .

Then, the hypothesis to be tested is:

An optimally conceived organization shows a dimensionality between 2,2 and 2,3.

This law should hold independent of size, sector or activity, or other situational factors (context). Small exceptions are conceivable: for example, in the case of an enduringly constant environment, a lower dimensionality would be thinkable as optimal, but probably only in economic terms. Note, however, that optimization in one dimension only, is in principle problematic in complex systems: Whenever one variable in a complex system is maximized, the likelihood increases that bottlenecks will spread and that consequently non-stable or chaotic behavior will ensue [2].

\section{Test of Hypothesis}

The hypothesis to be tested is that:

An optimally conceived organization shows a dimensionality between 2,2 and 2,3.

There are several ways of testing this hypothesis. The usual one would be to proceed with a comparison, scrutinizing a number of organizations in similar situations, measuring a) their performance and b) their organizational dimensionality, and subsequently examining whether the structure-performance link implied by this hypothesis is corroborated by the data.

A second approach would be to test whether a structural arrangement already proven or at least considered optimal is in accordance with the hypothesis.

Even though the first test would be the stronger option, initially the second one is to be carried out, as it involves a lower cost.

\subsection{Setup}

The setup will provide for examining a theory for an optimal structuring of interactions and communications in large groups, - the Team Syntegrity model (TSM). This is a structural framework to foster cohesion and synergy in larger groups of individuals, or to encourage the transformation of mere aggregates of individuals with similar interests into organizations with their own identities. Invented by Stafford Beer [7], TSM is a progressive design for democratic management in the sense of the heterar- 
chical-participative type of organization [cf. 20,21]. The TSM is a holographic model for organizing processes of communication in a non-hierarchical fashion that can be shown to be mathematically optimal for the (self-) management of social systems. Based on the structure of polyhedra, it is especially suitable for realizing teamoriented structures, and for supporting processes of planning, knowledge generation and innovation in turbulent environments. In the following, I shall illustrate the architecture of the model by using the geometry of an icosahedron, which is one of the structures commonly used to organize syntegration events, - in this case with 30 participants.

The formation of networks by persons who are connected by mutual interests is a manifestation of the information/knowledge society and a structural answer to challenges of our time. An infoset is a set of individuals who share a common concern and who are in possession of pertinent information or knowledge connected with the issues of concern, and who have the will (and most likely also the enthusiasm) to tackle these. The Team Syntegrity model supplies the structural framework for the synergetic interaction of an infoset which is intended to lead to an integration of multiple topics and perspectives towards a shared body of knowledge. The term Syntegrity results from a combination of synergy and tensile integrity. We speak of synergy when the interaction or co-operation of two or more agents produces a combined effect greater than the sum of their individual efforts. Tensile integrity is the structural strength provided by tension, as opposed to compression [9].

\subsection{Structural Architecture of Team Syntegrity}

An infoset of 30 persons, for example, can organize itself according to the structure of an icosahedron, the most complex of the regular, convex polyhedra (Fig. $1^{3}$ ), whereas for smaller gatherings, structures based on other polyhedra are possible. Each member of the a 30 member infoset is represented by one edge on the icosahedron. Each vertex stands for a team of five players ( $\rightarrow$ five edges) working on one topic; in an icosahedron there are 12 vertices that would be marked by different colors in a Syntegration. Therefore, given the geometry, each participant as a player/actor is connected by his/her edge to two different teams. Ms. Red-Yellow, for instance, belongs to the teams (vertices) Red and Yellow. At the same time, she acts as a critic to two other teams (for example, Black and Silver, which are her immediate neighbors). This means that each team consists of 5 players and 5 critics. Altogether, the thirty agents perform a total of 120 roles ( 30 times 2 roles as a player and 2 as a critic). In addition, there is the observer role, which will be explained in a moment.

This structure resolves the paradox of peripherality versus centrality of actors in an organization (as formalized by Bavelas [3]): While peripherality leads to communication pathologies, alienation and low morale, centrality is needed for effective action. However, as a group grows, centrality can only be ,purchased" at the cost of increasing peripherality [16]. Team Syntegrity enables an Infoset to acquire "centrality“ via a reverberative process (each team will meet more than once), although the peripherality of each one of its members equals zero, i.e., there is no peripherality at all.

\footnotetext{
${ }^{3}$ Kindly made available by TSI - Team Syntegrity Inc., Toronto, Canada.
} 


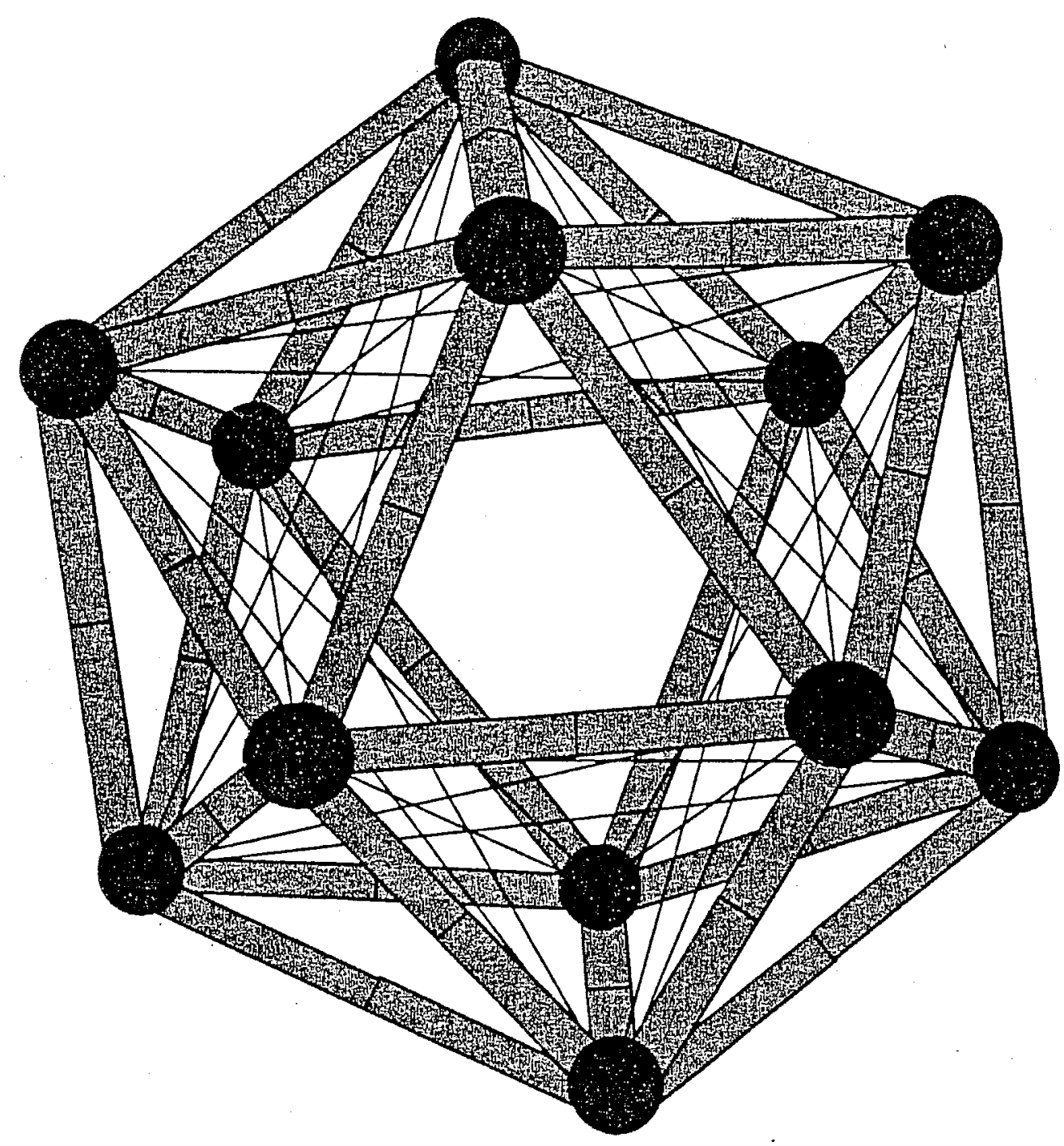

Fig. 1. Icosahedral structure of the Team Syntegrity model

Typically, the structure of Team Syntegrity is applied in the context of process by which ill-defined issues have to be tackled and for which contributions of multip agents with different backgrounds are required. This is the case e.g. in strategy ma ing, knowledge generation and organizational development. A Syntegration proce has the following phases: After the phases of initialization and joint design of $t$ l agenda around the common subject of interest, the 12 individual teams (consisting 5 players and 5 critics each) explore their respective topic. Each team meets sever (usually three) times and writes up a summary of its results to share with the who infoset. Discussions evolve as follows: The sessions are developed in a parallel mor with two teams working at a time. This means that 20 of the 30 members of the infi set are involved in these discussions. The remaining 10 can attend any one of th meetings as observers, in order to complement the views derived from their activitit as players and critics in their respective, individual set of 4 teams. They may also u: some of that time for lateral conversations with other "idle" members or simply relax 
The fact that the same issue with its different but interconnected aspects is continually processed by the same set of people, who gather in alternating compositions (topic-oriented teams). implies strong reverberation and leads to a self-organizing process with high levels of knowledge integration. There is no center required to integrate the individual efforts; integration just occurs of its own accord. It can be shown mathematically that this is a geometrically ergodic process, in which the eigenvalue 4 of the process converges to a minimum: Ninety percent of the information in the system will be shared after three iterations, and ninety-six after four iterations [13].

\subsection{Calculus of the Dimensionality of the Team Syntegrity Architecture}

In the following, a rough calculus of the dimensionality of an infoset as structured by the icosahedral architecture of Team Syntegrity ensues:

$$
R_{l}=R_{T}+R_{C}
$$

where $R_{I}$ denotes the total set of actual relationships of the members of the infoset. Its components are $R_{T}$, - the relationships at the team level, and $R_{C}$, - the complementary relationships of the observers.

$R_{T}$ is the composite of the relationships within the teams $(t), 12$ in the ideal case. $n_{i}$ expresses the number of team members of team $i$, the ideal number of members being five players $(p)$ plus five critics $(c)$ for all teams. The number of relationships between a pair of members is denoted by $(m)$.

$$
R_{T}=\sum_{i=1}^{l} \frac{m \cdot n_{i}\left(n_{i}-1\right)}{2}, n_{i}=p_{i}+c_{i} .
$$

For this ideal case, with reciprocal relationships between each pair of members, i.e. $(\mathrm{m}=2), R_{T}$ amounts to:

$$
R_{T}=12 \times 2 \times 10 \times 9 \times 1 / 2=1^{\prime} 080
$$

$R_{C}$ is the composite of the relationships between the members of the discussing teams $(b)$ and the members of the team they observe. The arrangement is that, while team discussions are going on, some of the observers relax ${ }^{5}$ whereas others switch from team to team, visiting both sessions going on at the time. Switches can also be made between the iterations of the team discussions, i.e., an observer could distribute his activities between the three iterations: For example, in the three iterations of the parallel discussions of teams $A$ and $B$ he or she could observe team $A$ in the first iteration, relax in the second, and observe team $B$ in the third iteration. To take account of these aspects, some assumptions must be made explicit to arrive at a first, rough calculation. We establish a parameter $f$ denoting the average percentage of the

${ }^{4}$ The formula to calculate the eigenvalue is: $y=(1 / \sqrt{5})^{\mathrm{n}}$, with $\mathrm{n}$ denoting the number of iterations.

s Such relaxation is essential to keep the vigor, concentration and involvement of participants high. 
total number of observers $b$ (ideally 10 ) which are actively observing teams during given pair of sessions. Furthermore, we introduce a parameter $s$ which expresses th average fraction of those active observers who switch between teams. Based on th many Syntegration events realized to date, including those accompanied by th author ${ }^{6}$, the assumption of $f=1 / 2$ and $s=1 / 3$ appears to be realistic for a rough ap proximation of an idealized Syntegration 7 .

Consequently, the following formula can be applied:

$$
R_{C}=\sum_{i=1}^{t} b \cdot n \cdot f \cdot(1+s)
$$

For this ideal-type we get:

$$
R_{C}=12 \times 10 \times 10 \times 1 / 2(1+1 / 3)=800
$$

Adding up $R_{T}$ and $R_{C}$ leads to:

$$
R_{I}=1^{\prime} 080+800=1^{\prime} 880
$$

In other words, the set of relationships of the icosahedral infoset, as specifier above, totals 1'880. At this point, the dimensionality of the structure $(x)$ can be cal culated as a function of $R_{I}$ and the total number of the members of the infoset $(N)^{8}$ :

$$
R_{1}=N^{x}
$$

To solve this equation the following transformations are necessary:

$$
\begin{gathered}
\log R_{I}=x \log N, \\
x=\frac{\log R_{I}}{\log N} .
\end{gathered}
$$

With a total set of relationship $\left(R_{l}\right)$ of 1'880 and a total number of infoset members $(N)$ of 30 , the result is:

${ }^{6}$ To date approximately 150 Syntegrations have been realized, despite the relative recency of the model. The author has directed or co-directed several, among them the first worldwide electronic Syntegration [cf. 8] and accompanied many more, within the framework of a research association with Stafford Beer, the creator of the model, and TSI-Team Syntegrity Inc., Toronto, the organization which makes Team Syntegrity available to organizations.

${ }^{7}$ The assumptions made explicit here try to capture a structure which enables an "optimal" flow of information, taking into account the psycho-physically limited resilience of participants. Variations of the parameters $f$ and $s$ as a function of the situation at hand should also be considered (see below).

${ }^{8}$ Equation (6) is isomorphic with equation (3) in section 2 . Therefore, $N$ is formally identical with the $L$, and $R_{I}$ with the $M$, in the latter. In other words, an isomorphic correspondence between Length and Number of members of an Infoset, as well as between Metabolic Rate and Number of Actual Relation- ships between Infoset Members is assumed. 


$$
x=\frac{\log 1 ' 880}{\log 30}=2,21658 .
$$

In other words, the dimensionality of the icosahedral architecture of Team Syntegrity is 2,21658 . In sum, the working hypothesis formulated above is strongly corroborated.

The surprising fact is that this size of $x$ is very close to the optimal dimensionality observed in biological organisms. It is actually closer to 2,22 than originally expected (cf. hypothesis above).

\subsection{The Revised Theorem}

In the light of these results, the hypothesis formulated above can be slightly revised, in the sense of proposing the following Theorem for an Optimal Structure of Organizations:

An optimal organization structure shows a dimensionality of approximately 2,22.

\subsection{Discussion}

I am aware that this proposition is bold, but it conforms to Popper's principle of falsifiability. In principle, this Theorem for an Optimal Structure of Organizations provides a powerful conceptual instrument to establish whether the dimensionality of a structure is too high or too low. The benefit lies in avoiding potentially huge costs and a host of disfunctionalities, - not only economic but also social and ecological ones.

However, the theorem also prompts questions. One major question that emerges is, how general this theorem is. Does not contingency theory postulate that organizational structures are and should be a function of the contexts they face? According to contingency theory, placid environments require and induce less complex structures than turbulent ones [cf. 15, 27]. The answer is straightforward: The theorem proposed here defines optimality in terms of contexts similar to those faced by living biological organisms. These are always confronted with complex, turbulent environments, at least potentially. Also, in the social domain potential high-level complexity and turbulence are ubiquitous.

Team Syntegrity, the reference model used for the test above, is definitely a model to be recommended for dealing with complex issues, but it would not be advisable for the structuring of a mere routine task. In addition, coping with that kind of task would most probably not require an organization of a dimensionality of 2,22 . However, routine tasks are usually part of more encompassing organizations, which in the end strive for viability and development $[22,23]$. As a whole, these organizations are in principle exposed to high complexity, at least potentially.

Further research should explore the possible limits of this theorem. Admittedly, a limitation of this paper is one of extension: Therefore, not all the practical implications, which are already discernible at this point, can be treated in detail.

For example, this first test has been confined to one organizational model, albeit under consideration of multiple modalities of its use. Other models for organizational 
structuring, which cannot be examined here, should be studied in the light of this Theorem for an Optimal Structure of Organizations. Also, the test applied here, has essentially been realized in a deductive mode. In addition, empirical tests of the type mentioned at the beginning of Section 3 should be carried out in the future.

Furthermore, variants of the assumptions underlying formula (5) of the calculus should be considered ${ }^{9}$. For example, possible trade-offs between parameters $f$ and $s$ should be studied; see also the sensitivity analysis in the following section. Finally, a great deal could be gained by improving and fine-tuning organizational models and methodologies, - Team Syntegrity being one of them -, in the light of this theorem.

\section{Sensitivity Analysis}

Following up on the discussions of the calibration of parameters, a number of scenarios were calculated in order to test the sensitivity of the TSM structure's dimensionality to changes in these parameters. A summary is presented in Table 1.

Table 1: Sensitivity Analysis - Summary

\begin{tabular}{|c|c|c|c|c|c|c|c|c|c|c|c|c|c|c|}
\hline Scenarios & \begin{tabular}{|l|} 
No. \\
Rela- \\
tion- \\
ships \\
betw. \\
Pairs \\
\end{tabular} & $\begin{array}{l}\text { No. } \\
\text { Play- } \\
\text { ers } \\
\end{array}$ & $\begin{array}{l}\text { No. } \\
\text { Cri- } \\
\text { tics } \\
\end{array}$ & $\begin{array}{l}\text { No. } \\
\text { Mem- } \\
\text { bers } \\
\text { Team } \\
\end{array}$ & $\begin{array}{l}\text { No. } \\
\text { Teams } \\
\end{array}$ & \begin{tabular}{|l|} 
No. \\
Rela- \\
tion- \\
ships \\
Team \\
Level \\
\end{tabular} & $\begin{array}{l}\text { No. } \\
\text { Ob- } \\
\text { ser- } \\
\text { vers }\end{array}$ & $\begin{array}{l}\text { Active } \\
\text { Ob- } \\
\text { servers }\end{array}$ & $\begin{array}{l}\text { Share } \\
\text { Swit- } \\
\text { chers }\end{array}$ & \begin{tabular}{|l|} 
No. \\
Compl. \\
Rel. \\
Ships \\
(Obser- \\
vers) \\
\end{tabular} & \begin{tabular}{|l} 
Total \\
No. \\
Rela- \\
tion- \\
ships \\
\end{tabular} & $\begin{array}{l}\text { Dimen- } \\
\text { siona- } \\
\text { lity }\end{array}$ & $\begin{array}{l}\text { Differen- } \\
\text { ce from } \\
\text { ideal }\end{array}$ & tion \\
\hline & $\mathrm{m}$ & $\mathrm{p}$ & c & $n$ & $i i$ & RT & b & $f$ & $s$ & RC & RI & D & $\mathrm{D}=2,22$ & \\
\hline Base & 2 & 5 & 5 & 10 & 12 & 1080 & 10 & 0.500 & 0.333 & 800 & 1880 & 2.21658 & -0.00342 & \\
\hline $\begin{array}{l}\text { Incomplete } \\
\text { Teams }\end{array}$ & 2 & 3 & 2 & 6 & 12 & 360 & 6 & 0.500 & 0.333 & 288 & 648 & 2.23981 & 0.01981 & \\
\hline Less Teams & 2 & 5 & 5 & 10 & is & 810 & 7.5 & 0.500 & \begin{tabular}{|l|}
0.333 \\
\end{tabular} & 450 & 1260 & 2.29286 & 0.07286 & $\frac{0.5}{3.2}$ \\
\hline $\begin{array}{l}\text { Non"- } \\
\text { Communica- } \\
\text { tors }\end{array}$ & 1.5 & 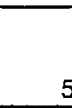 & 5 & 10 & 12 & 810 & 10 & 0.500 & 0.333 & 800 & 1610 & 2.17100 & -0.04900 & -2 \\
\hline Work & 2 & 7 & 5 & 10 & 12 & 1080 & 10 & (18:30 & 2.860 & 1728 & 2808 & 2.33454 & 0.11454 & 5. \\
\hline Lazybones & 2 & 5 & 5 & 10 & 12 & 1080 & 10 & 320 & (1,.20\% & 288 & 1368 & 2.12311 & -0.09689 & -4 \\
\hline
\end{tabular}

The scenarios are:

1. Base: This scenario corresponds to the "ideal case" as in the calculations above.

2. Incomplete Teams: Parameters $p$ and $c$, which denote the numbers of players and critics per team are set to 3 respectively, instead of 5 , as in the base scenario.

3. Less Teams: Parameter $i$ which represents the number of teams is reduced from 12 to 9 .

${ }^{9}$ Empirical studies will - ceteris paribus (all other factors being equal) - show different values for $f$ and $s$ depending on the circumstances of the respective Syntegration event: For example, a Syntegration with obligatory participation and limited commitment of participants tends to exhibit lower values for $f$ and for $s$, than the ones chosen in the calculus above. The opposite - higher values for $f$ and for $s$ - will tendentially be the case in a Syntegration of a group of people tackling a difficult issue all of them are highly committed to. 
4. "Non"-communicators: The average number of relationships between each pair of members of the infoset - captured by parameter $m$ - is reduced from 2 to 1,5 .

5. Workaholics: The share of active observers - denoted by parameter $f$ - and the share of those who switch teams in a given session $-s-$ are drastically increased.

6. Lazybones: : The share of active observers - $f$ - and the share of those who switch teams in a given session $-s-$ are drastically reduced.

The results show deviations between $0,15 \%$ and $5,16 \%$ from the ideal of $D=2,22$. The Team Syntegrity structure appears to be very robust against incompleteness of teams, weak communicators and even reduced team numbers. While the deviation of plus $5,16 \%$ in the case of the "Workaholics" scenario probably does not imply more than some unproductive work due to excessive activism, the deviation of minus $4,36 \%$ indicates that a low level of commitment may lead to some, albeit not even very strong decrease in the shared information. Altogether, it appears that it is difficult to be unproductive in the context of the Team Syntegrity structure.

\section{Synopsis and Outlook}

This paper has addressed the question of the optimality of organizational structures. First, the quest for optimality has been traced as observable throughout the endeavors of management science and organization theory. It has been shown that the pertinent research has come up with methods to identify optimal, or mostly close to optimal, solutions for specific problems. Theory has also provided models which define necessary prerequisites for viability (in the case of Living Systems Theory) and even sufficient structural preconditions for viability (in the case of the Viable Systems Model). However, the established body of knowledge has not furnished a general theorem, which would establish a norm for the dimensionality of optimally designed organizations.

Biological research into organic structures has empirically ascertained the fractal dimensionality of living organisms, which can be assumed to be optimal. Building on this body of knowledge, a new theorem for the design of optimal organizations has been proposed here. Also, a first test of the main proposition has been undertaken. The results suggest that the theorem is surprisingly accurate.

In addition to further testing of the proposition, follow-up research should address several important questions, two of which shall be pointed out here. The first question is: "What are operational measures of fractal dimensionalities, and how can they be achieved?" The second question is: "To what degree can the optimal dimensionality vary as a function of the properties of an organization, such as the cohesiveness or diversity of goals, values and preferences of its members?"

In sum, this Theorem for an Optimal Structure of Organizations is applicable to all kinds of social organisms, be they private firms, public organizations or social initiatives, etc. It opens up new prospects of a more rigorous assessment of models of structure proposed by theories of organization. But it also enables a better-founded evaluation of concrete structuring options, as well as a theory-based design and implementation of structural models in practice. In both contexts, this theorem offers a 
benchmark by means of which obsolete fads and fashions can be exposed and dis. functional propositions refuted. Finally, it must be emphasized that this theorem sheds new light on structural issues of the design of the structures by which a society gov. ems itself: The political system, the "state", i.e., government and the public sector ir general can benefit from it.

\section{Acknowledgements}

The author extends his thanks to Professor Anatol Rapoport and to Mr. Peter Vrhovec for reading drafts of this paper as well as for their valuable and encouraging comments. He is also very grateful to Mr. Heiko Eckert for making him aware of Sernetz' work and to Dr. Andreas Klose for reading parts of this paper and giving helpful technical advice. Finally, he thanks Professor Roberto Moreno-Diaz for providing an ideal working environment at the Institute of Cybernetics, University of Las Palmas de Gran Canaria, where most of this paper was written during a visiting professorship.

\section{References}

1. Ackoff, R.L./ Gharajedaghi J.: Mechanisms, Organisms and Human Systems. Strategic Management Joumal, Vol. 5, (1984) 289-300

2. Adam, A.: Modellplatonismus und Computerorakel. In: Wilhelm Bühler, et al., eds., Die ganzheitlich-verstehende Betrachtung der sozialen Leistungsordnung, Festschrift Josef Kolbinger, Wien/New York: Springer (1985)

3. Bavelas, A.: Communication Patterns in Problem Groups. In: Cybernetics: Transactions of the Eighth Conference, New York: Josiah Macy Jr. Foundation (1952)

4. Beer, S.: The Heart of Enterprise, Chichester etc.: Wiley (1979)

5. Beer, S.: Brain of the Firm, 2nd ed., Chichester etc.: Wiley (1981)

6. Beer, S.: Diagnosing the System for Organizations, Chichester etc.: Wiley (1985)

7. Beer, S.: Beyond Dispute. The Invention of Team Syntegrity, Chichester etc.: Wiley (1994)

8. Espejo, R./ Schwaninger, M., eds.: To Be and Not to Be that is the System, Festschrift für Stafford Beer, CD-ROM, Wiesbaden: Carl Auer Systeme Verlag (1998)

9. Fuller, R.B./ Applewhite, E.J.: Synergetics: Explorations in the Geometry of Thinking, New York/London: Macmillan/Collier (1982)

10. Garey, M. R./ Johnson, D. S.: Computers and Intractibility: A Guide to the Theory of NP. Completeness. Freeman, New York (1979)

11. Hausdorff, F.: Dimension und äusseres Mass. Mathematische Annalen 79 (1918) 157-179

12. Henschel, A.: Communities of Practice. Plattform für individuelles und kollektives Lemen sowie den Wissenstransfer, Doctoral Dissertation, Universität St. Gallen (2001; also to be published by Gabler Verlag, Wiesbaden)

13. Jalali, A.: Reverberating Networks. Modelling Information Propagation in Syntegration by Spectral Analysis. In: Beer, S., Beyond Dispute. The Invention of Team Syntegrity, Chichester etc.: Wiley (1994) 263-281

14. Jestczemski, F./ Bolterauer, H. / Sernetz, M.: Comparison of the Surface Dimension and the Mass Dimension of Blood Vessel Systems. In: Miyazima, S., ed.: The Future of Fractals, Singapore etc.: World Scientific (1997) 11-20 
15. Lawrence, P.R./Lorsch, J.W.: Organization and Environment: Managing Differentiation and Integration, Boston: Harvard University Press (1967)

16. Leonard, A.: Team Syntegrity: Planning for Action in the Next Century. In: Brady, B./ Peeno, L., eds., Proceedings, Conference of the International Society for the Systems Sciences, Louisville, Kentucky (1994) 1065-1072

17. Miller, J.G.: Living Systems, Niwot, Colorado: University Press of Colorado (1978; 1995 reprint)

18. Rapoport, A.: Fights, Games and Debates, Ann Arbor: University of Michigan Press (1960; 5th printing 1974)

19. Rapoport, A.: General System Theory, Essential Concepts and Applications, Tunbridge Wells, Kent /Cambridge, Mass.: Abacus Press (1986)

20. Schwaninger, M.: Structures for Intelligent Organizations, Discussion Papers, Institute of Management, University of St. Gallen, Switzerland, No. 20 (1996)

21. Schwaninger, M.: Distributed Control in Social Systems. In: Parra-Luna, F., ed.: The Performance of Social Systems, New York etc.: Kluwer Academic/Plenum Publishers (2000a) $147-173$

22. Schwaninger, M.: Managing Complexity - The Path Toward Intelligent Organizations. Systemic Practice and Action Research, Vol. 13, No. 2 (2000b) 207-241

23. Schwaninger, M.: Intelligent Organizations: An Integrative Framework. Systems Research and Behavioral Science, Vol. 18, Issue 2 (2001) 137-158

24. Sernetz, M.: Organisms as Open Systems. In: Nonnenmacher, T.F./ Losa, G.A./ Weibel, E.R., eds.: Fractals in Biology and Medicine, Basel etc.: Birkhäuser (1994) 232-239

25. Sernetz, M.: Die fraktale Geometrie des Lebendigen. Spektrum der Wissenschaft, July $2000,72-79$

26. Sernetz, M./ Justen, M./ Jestczemski, F.: Dispersive Fractal Characterization of Kidney Arteries by Three-Dimensional Mass-Radius-Analysis. In: Evertsz, C.J.G./ Peitgen, H.-O./ Voss, R.F., eds.: Fractal Geometry and Analysis. The Mandelbrot Festschrift, Curaçao 1995, Singapore etc.: World Scientific (1996) 475-487

27. Thompson, J.D.: Organizations in Action, New York etc.: McGraw-Hill (1967)

28. Wegener, I.: Theoretische Informatik. Eine algorithmenorientierte Einfuhrung, Stuttgart: Teubner (1993) 
Series Editors

Gerhard Goos, Karlsruhe University, Germany

Juris Hartmanis, Cornell University, NY, USA

Jan van Leeuwen, Utrecht University, The Netherlands

Volume Editors

Roberto Moreno-Díaz

Universidad de Las Palmas de Gran Canaria

Instituto Universitario de Ciencias y Tecnologías Cibernéticas

Campus de Tafira, 35017 Las Palmas de Gran Canaria, Spain

E-mail: rmoreno@ciber.ulpgc.es

Bruno Buchberger

University of Linz, Research Institute for Symbolic Computation

4232 Schloss Hagenberg, Austria

E-mail: Bruno.Buchberger@ risc.uni-linz.ac.at

José Luis Freire

University of A Coruña, Facultad de Informática, LFCIA, Dpto. of Computer Science

Campus de Elviña, 15071 A Coruña, Spain

E-mail: freire@fi.udc.es

Cataloging-in-Publication Data applied for

Die Deutsche Bibliothek - CIP-Einheitsaufnahme

Computer aided systems theory : a selection of papers from the 8th

International Workshop on Computer Aided Systems Theory, LasPalmas de

Gran Canaria, Spain, February 19 - 23, 2001 ; revised papers / EUROCAST 2001.

Roberto Moreno-Díaz ... (ed.). - Berlin ; Heidelberg ; New York ; Barcelona ;

Hong Kong ; London ; Milan ; Paris ; Tokyo : Springer, 2001

(Lecture notes in computer science; Vol. 2178)

ISBN 3-540-42959-X

CR Subject Classification (1998): J.6, I.6, I.2, J.7, J.3, C.1.m, C.3, F.4, F.3

ISSN 0302-9743

ISBN 3-540-42959-X Springer-Verlag Berlin Heidelberg New York

This work is subject to copyright. All rights are reserved, whether the whole or part of the material is concerned, specifically the rights of translation, reprinting, re-use of illustrations, recitation, broadcasting, reproduction on microfilms or in any other way, and storage in data banks. Duplication of this publication or parts thereof is permitted only under the provisions of the German Copyright Law of September 9, 1965, in its current version, and permission for use must always be obtained from Springer-Verlag. Violations are liable for prosecution under the German Copyright Law.

Springer-Verlag Berlin Heidelberg New York

a member of BertelsmannSpringer Science+Business Media GmbH

http://www.springer.de

(c) Springer-Verlag Berlin Heidelberg 2001

Printed in Germany

Typesetting: Camera-ready by author, data conversion by Boller Mediendesign

Printed on acid-free paper $\quad$ SPIN: $10840478 \quad 06 / 3142 \quad 543210$ 